\title{
Le bilan comparatif des médicaments : pas seulement un meilleur schéma thérapeutique possible
}

\author{
par Peter J Zed
}

$\mathrm{L}$ 'Institut pour la sécurité des médicaments aux patients du $\triangle$ Canada (ISMP Canada) définit le bilan comparatif des médicaments comme " un processus formel au cours duquel les professionnels de la santé travaillent de concert avec les patients, leur famille et les autres prestataires de soins de santé dans le but de s'assurer que des renseignements précis et exhaustifs sur les médicaments soient systématiquement communiqués à travers les interfaces de soins " ${ }^{1}$. Il est fondé sur " une analyse systématique et exhaustive de tous les médicaments pris par les patients pour s'assurer que les médicaments ajoutés, modifiés ou cessés soient évalués attentivement. Il représente une des composantes de la gestion des médicaments et permettra de fournir les renseignements permettant aux prescripteurs de prendre des décisions adéquates pour le patient ${ }^{1}$. Le bilan comparatif des médicaments est sanctionné par des organismes de sécurité des patients et mis en œuvre dans bon nombre d'établissements partout dans le monde afin de permettre une exactitude dans la communication au moment de l'admission, du transfert et du congé. Il a été introduit en 2005 dans le programme d'Agrément Canada à titre de Pratique organisationnelle requise. Ainsi, au cours de la dernière décennie, les établissements de santé ont été évalués en fonction de leur capacité à respecter ces normes, et on note que la conformité s'est améliorée dans l'ensemble du pays au cours de cette période ${ }^{2}$. Manifestement, le processus du bilan comparatif des médicaments est avantageux, et peu oseraient réfuter le fait qu'une meilleure communication au sujet des médicaments tout au long du continuum des soins permet de réduire les événements indésirables liés aux médicaments et d'améliorer l'observance thérapeutique du patient. De plus, mieux communiquer les renseignements touchant les médicaments au moment du transfert et du congé peut réduire le nombre de visites au service des urgences et de réadmissions imprévues à l'hôpital, permettant ainsi de diminuer considérablement l'utilisation des ressources en santé.
Obtenir le meilleur schéma thérapeutique possible (MSTP) au moment de l'admission représente une étape importante de la réalisation du bilan comparatif des médicaments. Bien que l'un des membres de l'équipe de pharmacie dresse normalement ce schéma, n'importe quel membre de l'équipe de soins de santé, voire un étudiant, peut l'établir correctement. D'ailleurs, des données probantes montrent que les pharmaciens et les autres fournisseurs de soins de santé peuvent obtenir avec exactitude le MSTP pour les patients au moment de l'admission, ce qui représente une étape menant à l'identification de différences relatives aux médicaments ${ }^{3}$. Or, des défis demeurent en ce qui touche à l'utilisation du MSTP en tant qu'élément du processus de gestion des médicaments et de la prestation de soins pharmaceutiques, une tâche beaucoup plus importante. En d'autres termes, l'obtention du MSTP est une étape essentielle, mais c'est bien l'évaluation et la résolution systématique des problèmes pharmacothérapeutiques ayant été décelés qui permettront aux patients d'obtenir de meilleurs résultats pour leur santé.

À ce jour, les ressources réservées à la réalisation de bilans comparatifs des médicaments sont principalement centrées sur l'admission alors que les transferts au sein d'un établissement de même que les congés sont plutôt négligés ${ }^{2}$. Les différences relatives aux médicaments présentes au moment du congé constituent notamment un problème aussi sérieux que les différences décelées à l'admission et elles risquent encore plus d'occasionner des événements indésirables si elles ne sont pas découvertes puis résolues ${ }^{4}$. En effet, jusqu'à $25 \%$ des patients connaissent un événement indésirable dans les 30 jours suivant leur congé et la moitié de ces cas pourraient être évités 5 . La réalisation méthodique d'un bilan comparatif des médicaments au moment du congé est l'un des moyens permettant d'éviter ces événements lorsqu'elle s'arrime au transfert des soins et à la communication avec les membres de l'équipe de soins du domaine communautaire ainsi 
qu'à une meilleure éducation des patients et de leur famille. Bien sûr, ce processus nécessite des ressources et des marches à suivre efficaces, mais il doit faire partie intégrante des soins aux patients.

Le processus qu'est le bilan comparatif des médicaments doit demeurer la pierre angulaire sur laquelle reposent les soins pharmaceutiques offerts à l'ensemble des patients, et ce, tout au long du continuum de leurs soins. Cependant, ce processus ne devrait pas s'arrêter aussitôt que l'on obtient le MSTP à l'admission. En effet, on devrait plutôt mettre l'accent sur l'identification et la résolution de tous les problèmes pharmacothérapeutiques pour tous les patients, alors que le MSTP est révisé et mis à jour à chaque étape du continuum des soins. Ce n'est qu'à l'aide de ces étapes importantes, par l'optimisation de la pharmacothérapie de chaque patient et la surveillance adéquate au moment de l'admission, du transfert et du congé, qu'il sera possible de réduire au maximum le nombre d'événements indésirables et de diminuer l'utilisation de ressources du système de santé, notamment en ce qui concerne les visites aux services des urgences et les hospitalisations imprévues. Obtenir le MSTP le plus précis à l'admission pour ensuite permettre à des problèmes pharmacothérapeutiques de demeurer en suspens tout au long du reste des transferts de soins ne profite à personne, et encore moins au patient. Il nous faut donc franchir la prochaine étape pour faire en sorte que le bilan comparatif des médicaments n'inclue pas seulement l'obtention du MSTP au moment de l'admission, mais qu'il comprenne aussi les meilleurs soins qu'il nous est possible d'offrir, le tout afin de faire place à la meilleure pharmacothérapie et à l'amélioration des résultats pour la santé qui en découleront sans conteste.

[Traduction par l'éditeur]
References

1. Bilan comparatif des médicaments $(B C M)$. Toronto $(\mathrm{ON})$ : Institut pour la sécurité des médicaments aux patients du Canada; (C) 2000-2015. Publié au : www.ismp-canada.org/fr/BCM.htm. Consulté le 2 janvier 2015.

2. Agrément Canada, Institut canadien d'information sur la santé, Institut canadien pour la sécurité des patients, Institut pour la sécurité des médicaments aux patients du Canada. Bilan comparatif des médicaments au Canada : hausser la barre. Progrès à ce jour et chemin à parcourir. Ottawa $(\mathrm{ON})$ : Agrément Canada; 2012.

3. Cornish PL, Knowles SR, Marchesano R, Tam V, Shadowitz S, Juurlink DN, et coll. Unintended medication discrepancies at the time of hospital admission. Arch Intern Med. 2005;165(4):424-9.

4. Wong JD, Bajcar JM, Wong GG, Alibhai SM, Huh JH, Cest A, et coll. Medication reconciliation at hospital discharge: evaluating discrepancies. Ann Pharmacother. 2008;42(10):1373-9.

5. Forster AJ, Clark HD, Menard A, Dupuis N, Chernish R, Chandok N, et coll. Adverse events among medical patients after discharge from hospital. CMAJ. 2004;170(3):345-9. Erratum publié dans CMAJ. 2004;170(5):771.

Peter J Zed, B. Sc., B.Sc. (Pharm.), ACPR, Pharm. D., FCSHP, est professeur agrégé et vice-doyen, programme Innovation de la pratique, Faculté des sciences pharmaceutiques, et membre associé, Département de médecine d'urgence, Faculté de médecine, Université de la Colombie-Britannique, Vancouver (Colombie-Britannique). II est également rédacteur adjoint $\mathrm{du} J \mathrm{CPH}$.

Intérêts concurrents : Aucun déclaré.

Address correspondence to:

Dr Peter J Zed

Faculty of Pharmaceutical Sciences

University of British Columbia

2405 Wesbrook Mall

Vancouver (BC) V6T 1 Z3

Courriel : peter.zed@ubc.ca

\section{CJHP Subscriptions 2015 / Abonnements au JCPH 2015}

New for 2015, we have moved to an online only version of CJHP, and have also introduced an institutional online subscription option. CJHP online is included as a benefit of CSHP membership. All prices are in Canadian funds.

En nouveauté pour 2015, nous avons migré vers la publication d'une seule version du JCPH, soit la copie électronique en ligne, et nous offrirons aussi en option un abonnement institutionnel. L'abonnement à la version électronique du JCPH publiée en ligne est inclus dans les droits d'adhésion à la SCPH. Tous les prix sont en dollars canadiens.

\begin{tabular}{ll}
$\begin{array}{l}\text { Subscriber group / } \\
\text { Groupe d'abonnés }\end{array}$ & $\begin{array}{l}\text { Individual Online Subscription / } \\
\text { Abonnement individuel en ligne }\end{array}$ \\
\hline Nonmembers within Canada / & $\$ 160.00$ per year, plus GST or HST \\
Non-membres au Canada & $160,00 \$$ par an, plus TPS ou TVH \\
\hline USA / É.-U. & $\$ 190.00$ per year \\
& $190,00 \$$ par an \\
\hline Foreign / Étranger & $\$ 230.00$ per year \\
& $230,00 \$$ par an
\end{tabular}

NEW Institutional Online Subscriptions / NOUVEL abonnement institutionnel en ligne

$\$ 480.00$ per year, plus GST or HST 480,00 \$ par an, plus TPS ou TVH

$\$ 510.00$ per year $510,00 \$$ par an $\$ 550.00$ per year $550,00 \$$ par an

More details can be found at www.cjhp-online.ca. Please direct comments or questions to cjhpedit@cshp.ca.

Des détails supplémentaires sont fournis à www.cjhp-online.ca. Pour tout commentaire ou toute question, veuillez vous adresser à cjhpedit@cshp.ca. 